\title{
Some improvements of Minkowski's integral inequality on time scales
}

\section{Guang-Sheng Chen*}

\section{"Correspondence:}

cgswavelets@126.com

Department of Construction and

Information Engineering, Guangxi

Modern Vocational Technology

College, Hechi, Guangxi 547000,

P.R. China

\begin{abstract}
In the paper, we establish some improvements of Minkowski's inequality on time scales via the delta integral, nabla integral and diamond- $\alpha$ dynamic integral, which is defined as a linear combination of the delta and nabla integrals.

MSC: 26D15; 26E70

Keywords: delta and nabla integrals; diamond- $\alpha$ integral; Minkowski's inequality; Hölder's inequality; time scales
\end{abstract}

\section{Introduction}

In 1988, Hilger in [1] established the theory of time scales in his doctoral dissertation, which resulted in his seminal paper in [2]. His work aimed to unify and generalize various mathematical concepts according to the theories of discrete and continuous analysis. Much information concerning time scales and dynamic equations on time scales can be found in the literature in [3-12]. Since then many authors have studied some integral inequalities on time scales in [13-16]. In [13, 14], the authors described the delta integral Minkowski's inequality on time scales as follows.

Theorem 1.1 Let $f, g, h \in C_{\mathrm{rd}}([a, b], \mathbb{R})$ and $1 / p+1 / q=1$ with $p>1$. Then

$$
\begin{aligned}
& \left(\int_{a}^{b}|h(x)||f(x)+g(x)|^{p} \Delta x\right)^{\frac{1}{p}} \\
& \quad \leq\left(\int_{a}^{b}|h(x)||f(x)|^{p} \Delta x\right)^{\frac{1}{p}}+\left(\int_{a}^{b}|h(x)||g(x)|^{p} \Delta x\right)^{\frac{1}{p}} .
\end{aligned}
$$

Nabla and diamond- $\alpha$ integral Minkowski's inequality on time scales was established in [15], which can be stated as follows.

Theorem 1.2 Let $f, g, h \in C_{\mathrm{ld}}([a, b], \mathbb{R})$ and $1 / p+1 / q=1$ with $p>1$. Then

$$
\begin{aligned}
& \left(\int_{a}^{b}|h(x)||f(x)+g(x)|^{p} \nabla x\right)^{\frac{1}{p}} \\
& \quad \leq\left(\int_{a}^{b}|h(x)||f(x)|^{p} \nabla x\right)^{\frac{1}{p}}+\left(\int_{a}^{b}|h(x)||g(x)|^{p} \nabla x\right)^{\frac{1}{p}} .
\end{aligned}
$$

(0) 2013 Chen; licensee Springer. This is an Open Access article distributed under the terms of the Creative Commons Attribution License (http://creativecommons.org/licenses/by/2.0), which permits unrestricted use, distribution, and reproduction in any medium, provided the original work is properly cited. 
Theorem 1.3 Let $f, g, h:[a, b] \rightarrow \mathbb{R}$ be $\diamond_{\alpha}$-integrable functions, and $1 / p+1 / q=1$ with $p>1$. Then

$$
\begin{aligned}
& \left(\int_{a}^{b}|h(x)||f(x)+g(x)|^{p} \diamond_{\alpha} x\right)^{\frac{1}{p}} \\
& \quad \leq\left(\int_{a}^{b}|h(x)||f(x)|^{p} \diamond_{\alpha} x\right)^{\frac{1}{p}}+\left(\int_{a}^{b}|h(x)||g(x)|^{p} \diamond_{\alpha} x\right)^{\frac{1}{p}} .
\end{aligned}
$$

Throughout paper, we suppose that $\mathbb{T}$ is a time scale, $a, b \in \mathbb{T}$ with $a<b$ and an interval $[a, b]$ means the intersection of a real interval with the given time scale. By a time scale $\mathbb{T}$, we mean an arbitrary nonempty closed subset of real numbers. The set of real numbers, integers, natural numbers, and the Cantor set are examples of time scales. But rational numbers, irrational numbers, complex numbers, and the open interval between 0 and 1 are not time scales.

The purpose of this paper is to establish some improvements of Minkowski's inequality by using the delta integral, the nabla integral and the diamond- $\alpha$ integral on time scales.

\section{Main results}

In this section, our main results are stated and proved.

Theorem 2.1 Let $f, g, h \in C_{\mathrm{rd}}([a, b], \mathbb{R}), p>0, s, t \in \mathbb{R} \backslash\{0\}$, and $s \neq t$. Let $p, s, t \in \mathbb{R}$ be different, such that $s, t>1$ and $(s-t) /(p-t)>1$. Then

$$
\begin{aligned}
& \int_{a}^{b}|h(x)||f(x)+g(x)|^{p} \Delta x \\
& \leq {\left[\left(\int_{a}^{b}|h(x)||f(x)|^{s} \Delta x\right)^{\frac{1}{s}}+\left(\int_{a}^{b}|h(x)||g(x)|^{s} \Delta x\right)^{\frac{1}{s}}\right]^{s(p-t) /(s-t)} } \\
& \times {\left[\left(\int_{a}^{b}|h(x)||f(x)|^{t} \Delta x\right)^{\frac{1}{t}}+\left(\int_{a}^{b}|h(x)||g(x)|^{t} \Delta x\right)^{\frac{1}{t}}\right]^{t(s-p) /(s-t)} . }
\end{aligned}
$$

Proof We have $(s-t) /(p-t)>1$, and in view of

$$
\begin{aligned}
& \int_{a}^{b}|h(x)||f(x)+g(x)|^{p} \Delta x \\
& \quad=\int_{a}^{b}|h(x)|\left(|f(x)+g(x)|^{s}\right)^{(p-t) /(s-t)}\left(|f(x)+g(x)|^{t}\right)^{(s-p) /(s-t)} \Delta x,
\end{aligned}
$$

by using Hölder's inequality in $[13,14]$ with indices $(s-t) /(p-t)$ and $(s-t) /(s-p)$, we have

$$
\begin{aligned}
& \int_{a}^{b}|h(x)||f(x)+g(x)|^{p} \Delta x \\
& \quad \leq\left(\int_{a}^{b}|h(x)||f(x)+g(x)|^{s} \Delta x\right)^{(p-t) /(s-t)}\left(\int_{a}^{b}|h(x)||f(x)+g(x)|^{t} \Delta x\right)^{(s-p) /(s-t)} .
\end{aligned}
$$


On the other hand, by using Minkowski's inequality (1.1) for $s>1$ and $t>1$, respectively, we obtain

$$
\begin{aligned}
& \left(\int_{a}^{b}|h(x)||f(x)+g(x)|^{s} \Delta x\right)^{\frac{1}{s}} \\
& \quad \leq\left(\int_{a}^{b}|h(x)||f(x)|^{s} \Delta x\right)^{\frac{1}{s}}+\left(\int_{a}^{b}|h(x)||g(x)|^{s} \Delta x\right)^{\frac{1}{s}},
\end{aligned}
$$

and

$$
\begin{aligned}
& \left(\int_{a}^{b}|h(x)||f(x)+g(x)|^{t} \Delta x\right)^{\frac{1}{t}} \\
& \quad \leq\left(\int_{a}^{b}|h(x)||f(x)|^{t} \Delta x\right)^{\frac{1}{t}}+\left(\int_{a}^{b}|h(x)||g(x)|^{t} \Delta x\right)^{\frac{1}{t}} .
\end{aligned}
$$

From (2.2), (2.3) and (2.4), this completes the proof of Theorem 2.1.

Remark 2.1 For Theorem 2.1, for $p>1$, letting $s=p+\varepsilon, t=p-\varepsilon$, when $p, s, t$ are different, $s, t>1$, and letting $\varepsilon \rightarrow 0$, we obtain (1.1).

Theorem 2.2 Let $f, g, h \in C_{\mathrm{rd}}([a, b], \mathbb{R}), p>0, s, t \in \mathbb{R} \backslash\{0\}$, and $s \neq t$. Let $p, s, t \in \mathbb{R}$ be different, such that $s, t<1$ and $s, t \neq 0$, and $(s-t) /(p-t)<1$. Then

$$
\begin{aligned}
\int_{a}^{b}|h(x)||f(x)+g(x)|^{p} \Delta x & \\
\geq & {\left[\left(\int_{a}^{b}|h(x)||f(x)|^{s} \Delta x\right)^{\frac{1}{s}}+\left(\int_{a}^{b}|h(x)||g(x)|^{s} \Delta x\right)^{\frac{1}{s}}\right]^{s(p-t) /(s-t)} } \\
\times & {\left[\left(\int_{a}^{b}|h(x)||f(x)|^{t} \Delta x\right)^{\frac{1}{t}}+\left(\int_{a}^{b}|h(x)||g(x)|^{t} \Delta x\right)^{\frac{1}{t}}\right]^{t(s-p) /(s-t)} . }
\end{aligned}
$$

Proof We have $(s-t) /(p-t)<1$, and in view of

$$
\begin{aligned}
& \int_{a}^{b}|h(x)||f(x)+g(x)|^{p} \Delta x \\
& \quad=\int_{a}^{b}|h(x)|\left(|f(x)+g(x)|^{s}\right)^{(p-t) /(s-t)}\left(|f(x)+g(x)|^{t}\right)^{(s-p) /(s-t)} \Delta x,
\end{aligned}
$$

by using reverse Hölder's inequality in $[13,14]$ with indices $(s-t) /(p-t)$ and $(s-t) /(s-p)$, we obtain

$$
\begin{aligned}
& \int_{a}^{b}|h(x)||f(x)+g(x)|^{p} \Delta x \\
& \quad \geq\left(\int_{a}^{b}|h(x)||f(x)+g(x)|^{s} \Delta x\right)^{(p-t) /(s-t)}\left(\int_{a}^{b}|h(x)||f(x)+g(x)|^{t} \Delta x\right)^{(s-p) /(s-t)} .
\end{aligned}
$$


On the other hand, in view of Minkowski's inequality (see [17]) for the cases of $s<1$ and $t<1$,

$$
\begin{aligned}
& \left(\int_{a}^{b}|h(x)||f(x)+g(x)|^{s} \Delta x\right)^{\frac{1}{s}} \\
& \quad \geq\left(\int_{a}^{b}|h(x)||f(x)|^{s} \Delta x\right)^{\frac{1}{s}}+\left(\int_{a}^{b}|h(x)||g(x)|^{s} \Delta x\right)^{\frac{1}{s}},
\end{aligned}
$$

and

$$
\begin{aligned}
& \left(\int_{a}^{b}|h(x)||f(x)+g(x)|^{t} \Delta x\right)^{\frac{1}{t}} \\
& \quad \geq\left(\int_{a}^{b}|h(x)||f(x)|^{t} \Delta x\right)^{\frac{1}{t}}+\left(\int_{a}^{b}|h(x)||g(x)|^{t} \Delta x\right)^{\frac{1}{t}} .
\end{aligned}
$$

Combining (2.6), (2.7) and (2.8), this completes the proof of Theorem 2.2.

Theorem 2.3 Let $f, g, h \in C_{\mathrm{ld}}([a, b], \mathbb{R}), p>0, s, t \in \mathbb{R} \backslash\{0\}$, and $s \neq t$. Let $p, s, t \in \mathbb{R}$ be different, such that $s, t>1$ and $(s-t) /(p-t)>1$. Then

$$
\begin{aligned}
& \int_{a}^{b}|h(x)||f(x)+g(x)|^{p} \nabla x \\
& \leq\left[\left(\int_{a}^{b}|h(x)||f(x)|^{s} \nabla x\right)^{\frac{1}{s}}+\left(\int_{a}^{b}|h(x)||g(x)|^{s} \nabla x\right)^{\frac{1}{s}}\right]^{s(p-t) /(s-t)} \\
& \quad \times\left[\left(\int_{a}^{b}|h(x)||f(x)|^{t} \nabla x\right)^{\frac{1}{t}}+\left(\int_{a}^{b}|h(x)||g(x)|^{t} \nabla x\right)^{\frac{1}{t}}\right]^{t(s-p) /(s-t)} .
\end{aligned}
$$

Proof This proof is similar to the proof of Theorem 2.1, so we omit it here.

Remark 2.2 For Theorem 2.3, for $p>1$, letting $s=p+\varepsilon, t=p-\varepsilon$, when $p, s, t$ are different, $s, t>1$, and letting $\varepsilon \rightarrow 0$, we get (1.2).

Theorem 2.4 Let $f, g, h \in C_{\mathrm{ld}}([a, b], \mathbb{R}), p>0, s, t \in \mathbb{R} \backslash\{0\}$, and $s \neq t$. Let $p, s, t \in \mathbb{R}$ be different, such that $s, t<1$ and $s, t \neq 0$, and $(s-t) /(p-t)<1$. Then

$$
\begin{aligned}
& \int_{a}^{b}|h(x)||f(x)+g(x)|^{p} \nabla x \\
& \geq\left[\left(\int_{a}^{b}|h(x)||f(x)|^{s} \nabla x\right)^{\frac{1}{s}}+\left(\int_{a}^{b}|h(x)||g(x)|^{s} \nabla x\right)^{\frac{1}{s}}\right]^{s(p-t) /(s-t)} \\
& \quad \times\left[\left(\int_{a}^{b}|h(x)||f(x)|^{t} \nabla x\right)^{\frac{1}{t}}+\left(\int_{a}^{b}|h(x)||g(x)|^{t} \nabla x\right)^{\frac{1}{t}}\right]^{t(s-p) /(s-t)} .
\end{aligned}
$$

Proof The proof of Theorem 2.4 is similar to the proof of Theorem 2.2, so we omit it here. 
Theorem 2.5 Let $f, g, h:[a, b] \rightarrow \mathbb{R}$ be $\diamond_{\alpha}$-integrable functions, $p>0, s, t \in \mathbb{R} \backslash\{0\}$, and $s \neq t$. Let $p, s, t \in \mathbb{R}$ be different, such that $s, t>1$ and $(s-t) /(p-t)>1$. Then

$$
\begin{aligned}
\int_{a}^{b}|h(x)||f(x)+g(x)|^{p} \diamond_{\alpha} x \\
\leq \\
\quad\left[\left(\int_{a}^{b}|h(x)||f(x)|^{s} \diamond_{\alpha} x\right)^{\frac{1}{s}}+\left(\int_{a}^{b}|h(x)||g(x)|^{s} \diamond_{\alpha} x\right)^{\frac{1}{s}}\right]^{s(p-t) /(s-t)} \\
\quad \times\left[\left(\int_{a}^{b}|h(x)||f(x)|^{t} \diamond_{\alpha} x\right)^{\frac{1}{t}}+\left(\int_{a}^{b}|h(x)||g(x)|^{t} \diamond_{\alpha} x\right)^{\frac{1}{t}}\right]^{t(s-p) /(s-t)} .
\end{aligned}
$$

Proof This theorem is a direct extension of Theorem 2.1 and Theorem 2.3, so we omit this proof here.

Remark 2.3 For Theorem 2.5, for $p>1$, letting $s=p+\varepsilon, t=p-\varepsilon$, when $p, s, t$ are different, $s, t>1$, and letting $\varepsilon \rightarrow 0$, we get (1.3).

Theorem 2.6 Let $f, g, h:[a, b] \rightarrow \mathbb{R}$ be $\diamond_{\alpha}$-integrable functions, $p>0, s, t \in \mathbb{R} \backslash\{0\}$, and $s \neq t$. Let $p, s, t \in \mathbb{R}$ be different, such that $s, t<1$ and $s, t \neq 0$, and $(s-t) /(p-t)<1$. Then

$$
\begin{aligned}
\int_{a}^{b}|h(x)||f(x)+g(x)|^{p} \diamond_{\alpha} x \\
\geq \\
\quad\left[\left(\int_{a}^{b}|h(x)||f(x)|^{s} \diamond_{\alpha} x\right)^{\frac{1}{s}}+\left(\int_{a}^{b}|h(x)||g(x)|^{s} \diamond_{\alpha} x\right)^{\frac{1}{s}}\right]^{s(p-t) /(s-t)} \\
\quad \times\left[\left(\int_{a}^{b}|h(x)||f(x)|^{t} \diamond_{\alpha} x\right)^{\frac{1}{t}}+\left(\int_{a}^{b}|h(x)||g(x)|^{t} \diamond_{\alpha} x\right)^{\frac{1}{t}}\right]^{t(s-p) /(s-t)} .
\end{aligned}
$$

Proof This theorem is a direct extension of Theorem 2.2 and Theorem 2.4, so we omit this proof here.

Remark 2.4 For $\alpha=1$, inequality (2.11) reduces to inequality (2.1), inequality (2.12) reduces to inequality (2.5). For $\alpha=0$, inequality (2.11) reduces to inequality (2.9), inequality (2.12) reduces to inequality (2.10). For $\mathbb{T}=\mathbb{R}$, the main results of this paper reduce to the results in [18].

Remark 2.5 The results of this paper can be given for more general time-scale integrals, for example, Lebesgue time-scale integrals in [19] or even multiple Lebesgue time-scale integrals in [20]. 


\section{References}

1. Hilger, S: Ein Maßkettenkalkül mit Anwendung auf Zentrumsmannigfaltigkeiten. PhD thesis, Universität Würzburg (1988)

2. Hilger, S: Analysis on measure chains - a unified approach to continuous and discrete calculus. Results Math. 18 18-56 (1990)

3. Agarwal, RP, Bohner, M: Basic calculus on time scales and some of its applications. Results Math. 35(1-2), 3-22 (1999)

4. Atici, FM, Guseinov, GS: On Green's functions and positive solutions for boundary value problems on time scales. J. Comput. Appl. Math. 141, 75-99 (2002)

5. Bohner, M, Peterson, A: Dynamic Equations on Time Scales: An Introduction with Applications. Birkhäuser, Boston (2001)

6. Bohner, M, Peterson, A: Advances in Dynamic Equations on Time Scales. Birkhäuser, Boston (2002)

7. Sheng, Q, Fadag, M, Henderson, J, Davis, JM: An exploration of combined dynamic derivatives on time scales and their applications. Nonlinear Anal., Real World Appl. 7, 395-412 (2006)

8. Ammi, MRS, Ferreira, RAC, Torres, DFM: Diamond- $\alpha$ Jensen's inequality on time scales. J. Inequal. Appl. 2008, Article ID 576876 (2008). doi:10.1155/2008/576876

9. Rogers, JW Jr., Sheng, Q: Notes on the diamond- $\alpha$ dynamic derivative on time scales. J. Math. Anal. Appl. 326(1), 228-241 (2007)

10. Malinowska, AB, Torres, DFM: On the diamond-alpha Riemann integral and mean value theorems on time scales. Dyn. Syst. Appl. 18(3-4), 469-482 (2009)

11. Adıvar, M, Bohner, EA: Halanay type inequalities on time scales with applications. Nonlinear Anal. 74(18), 7519-7531 (2011)

12. Erbe, L: Oscillation criteria for second order linear equations on a time scale. Can. Appl. Math. Q. 9(4), 345-375 (2001)

13. Wong, FH, Yeh, CC, Yu, SL, Hong, CH: Young's inequality and related results on time scales. Appl. Math. Lett. 18, 983-988 (2005)

14. Wong, FH, Yeh, CC, Lian, WC: An extension of Jensen's inequality on time scales. Adv. Dyn. Syst. Appl. 1(1), 113-120 (2006)

15. Özkan, UM, Sarikaya, MZ, Yildirim, H: Extensions of certain integral inequalities on time scales. Appl. Math. Lett. 21(10), 993-1000 (2008)

16. Anwar, M, Bibi, R, Bohner, M, Pečarić, J: Integral inequalities on time scales via the theory of isotonic linear functionals. Abstr. Appl. Anal. 2011, Article ID 483595 (2011)

17. Kuang, JC: Applied Inequalities. Shandong Science and Technology Press, Jinan (2010)

18. Zhao, CJ, Cheung, WS: On Minkowski's inequality and its application. J. Inequal. Appl. 2011, 71 (2011)

19. Guseinov, GS: Integration on time scales. J. Math. Anal. Appl. 285, 107-127 (2003)

20. Bohner, M, Guseinov, GS: Multiple Lebesgue integration on time scales. Adv. Differ. Equ. 2006, Article ID 26391 (2006)

doi:10.1186/1029-242X-2013-318

Cite this article as: Chen: Some improvements of Minkowski's integral inequality on time scales. Journal of Inequalities and Applications 2013 2013:318.

\section{Submit your manuscript to a SpringerOpen ${ }^{\circ}$ journal and benefit from:}

- Convenient online submission

Rigorous peer review

- Immediate publication on acceptance

Open access: articles freely available online

- High visibility within the field

- Retaining the copyright to your article 